\title{
OMAE2019-95607
}

\section{HYDRODYNAMICS AROUND A DEEP-DRAFT SEMI-SUBMERSIBLE WITH BIOMIMETIC TUBERCLE CORNER DESIGN}

\author{
Yibo Liang', Weichao Shi, Longbin Tao. \\ Department of Naval Architecture, Ocean \& Marine Engineering \\ University of Strathclyde \\ Glasgow, G4 0LZ, UK
}

\section{ABSTRACT}

Leading-edge tubercles have been investigating widely on the performance of foils in the last decade. In this study, the biomimetic tubercle design has been applied to the corner shape on a deep-draft semi-submersible. A numerical study on flow over a deep-draft semi-submersible (DDS) with a biomimetic tubercle corner shape was carried out to investigate the corner shape effects on the overall hydrodynamics and motion responses. The hydrodynamic performance of the biomimetic tubercle corner is compared with a traditional round corner design platform. It is demonstrated that, as the corner shape design changed, the motion responses alter drastically. In addition, the flow patterns were examined to reveal some insights into fluid physics due to the biomimetic tubercle corner design. The comprehensive numerical results showed that the three-dimensional effect, which causes spanwise flow, can be reduced by a continuous spanwise (column-wise) variation of the shear-layer separation points.

Keywords: Vortex-Induced Motions, Semi-Submersible, Biomimetic Tubercle.

\section{NOMENCLATURE}

$\begin{array}{ll}A_{m} / L & \text { Non dimensional maximum motion amplitude } \\ A_{x} / L, A_{y} / L & \text { Non dimensional motion amplitude } \\ B_{L} & \text { Overall width of the structure } \\ B_{T} & \text { Draft of the structure } \\ \bar{C}_{D} & \text { Mean drag coefficient } \\ C_{L r m s} & \text { Root mean square lift coefficient } \\ D & \text { Projected length } \\ H & \text { Immersed column height above the pontoon } \\ L & \text { Column width }\end{array}$

$\begin{array}{ll}P & \text { Pontoon height } \\ R & \text { Corner radius } \\ S & \text { Distance between centre columns } \\ T_{0} & \text { Natural period of the structure in calm water } \\ U r & \text { Reduce velocity }\end{array}$

\section{INTRODUCTION}

The suppression of vortex-induced motions (VIM) of bluff bodies (offshore floating platforms, riser pipes etc.) raises a technological challenge within the offshore oil \& gas discipline. The fundamental fluid physics behind the phenomena attracts many researchers to investigate. For a cylindrical body, the helical strake is the most common technology employed by the industry to suppress vortex-induced vibrations (VIV). In the offshore engineering discipline, the helical strake significant extends the transportation and installation process for a floating platform (e.g. Spar). The researcher hence started to discover alternative ways to suppress VIV/VIM. Assi and Bearman [1] investigated the vortex-induced vibration of a wavy elliptic cylinder. Their study showed that the wavy elliptic cylinder does not eliminate VIV. Darekar and Sherwin [2] studied the flow past a square-section cylinder with a wavy stagnation face. It demonstrated that the drag force on a wavy square cylinder is constantly lower than that of a straight cylinder. A large number of studies have been carried out to understand the effect of corner shape designs, such as Delany and Sorensen [3], Bearman, et al. [4], Tamura and Miyagi [5]. Liang and Tao [6] also performed a study of the corner shape effects on a semi-submersible ${ }^{2}$.

Recently, the leading-edge tubercles on the pectoral fins of humpback whales have attracted the attention of researchers who wish to exploit this feature in the design of hydrofoil to improve the fluid dynamic performance [7]. A number of experimental

${ }^{1}$ Contact author: yibo.liang@strath.ac.uk

${ }^{2}$ Correction: The y label appeared on Figure 7 [6] should be $0.50,0.70,0.90,1.10,1.30$ and 1.50, instead of 0.30, $0.50,0.70,0.90$, and $1.10[6]$. 
and numerical studies on the leading-edge tubercles have been conducted in the last decade to understand the insight of the concept [8-12]. It demonstrated that the leading-edge undulation will excite the streamwise contra-rotating vortices which can energize the flow and accelerate the flow transition [13].

In the present study, a numerical study on flow over a deepdraft semi-submersible (DDS) with a biomimetic tubercle corner shape was carried out to investigate the corner shape effects on the overall hydrodynamics and motion responses. In addition, the hydrodynamic performance of the biomimetic tubercle corner is compared with a traditional rounded corner design. This paper mainly discusses the hydrodynamic loads on the structures, the motion responses of the platform as well as the flow patterns around the platform.

\section{METHODOLOGY}

In the present section, the characteristics of the benchmark DDS, tubercle corner design are introduced following with an overview of the numerical simulation.

\section{Model Characteristics}

The benchmark model (a DDS, with a scale ratio of 1:64 from prototype) is defined the same as previous outcomes from Liang, et al. [14]. Details are presented in Figure 1 and Table 1. The mooring lines arrangement is presented in Figure 2.

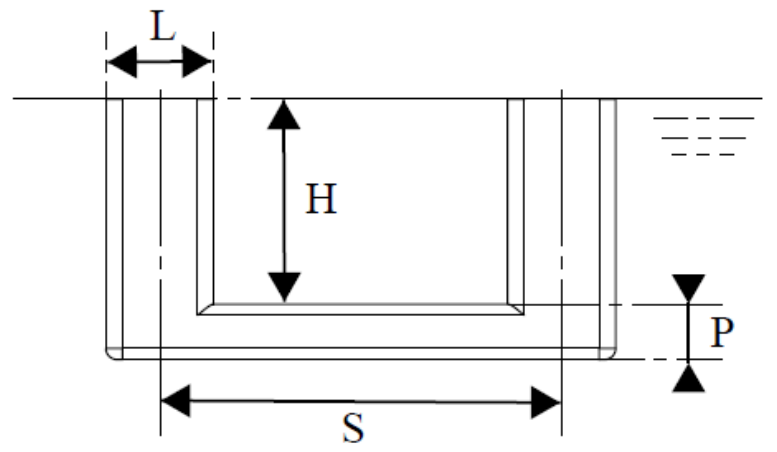

Figure 1. Benchmark DDS model presented in Ref [14].

In the present study, the corner shape is further modified with a tubercle design. The sinusoidal corner-shape profile (compared with the benchmark DDS) was developed as shown in the APPENDIX A. The tubercles are simulated as a sinusoidal corner-shape with corner ratio varying from $2 \%$ to $32 \%$.

\section{Computational Overview}

The flow fields have been numerical simulated using the detached eddy simulation (DES). For the DES model, the improved delayed detached eddy simulation (IDDES) model [15] with the Spalart-Almaras (SA) [16] was used. All the simulations were carried out using a commercial CFD code, STAR-CCM+ 13.04.011 [17]. The finite volume method (FVM) is adopted to discretize the incompressible flow field equations [17]. The second-order implicit three time levels (ITTL) scheme is applied for the temporal discretization. The convective term is evaluated by using a hybrid second-order upwind scheme.
SIMPLE algorithm is employed to treat the pressure and velocity coupling.

Table 1. Benchmark model characteristics.

\begin{tabular}{lc}
\hline & $\mathbf{1 : 6 4}$ Model $\quad(\mathbf{m})$ \\
\hline $\begin{array}{l}\text { Distance between centre columns } \\
(\boldsymbol{S})\end{array}$ & 1.133 \\
Column width $(\boldsymbol{L})$ & 0.305 \\
Immersed column height above the & 0.578 \\
pontoon $(\boldsymbol{H})$ & 0.156 \\
Pontoon height $(\boldsymbol{P})$ & 0.047 \\
Corner radius $(\boldsymbol{R})$ & $15 \%$ \\
Corner ratio $(\boldsymbol{R} / \boldsymbol{L})$ & \\
\hline
\end{tabular}

\section{Current direction}

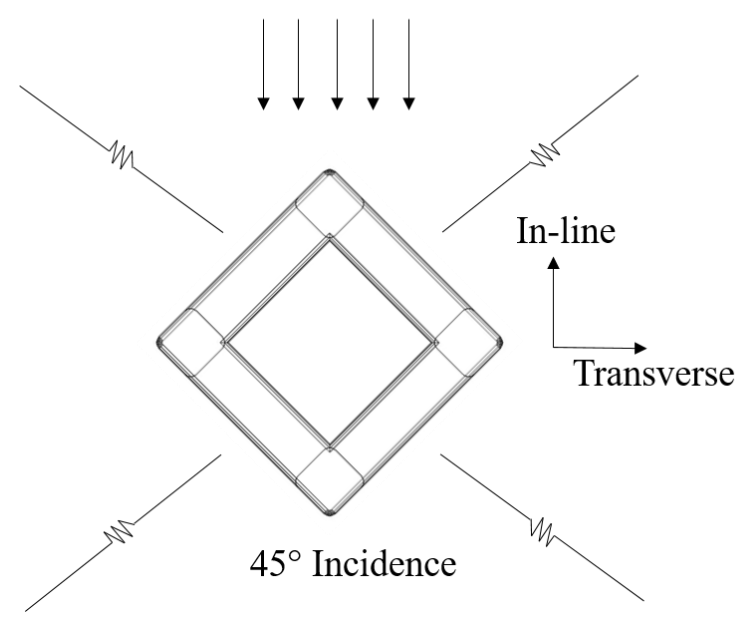

Figure 2. Mooring lines arrangement for the benchmark model.

The computational domain size, mesh and time step employed in the present numerical study were chosen based on previous experience with modelling vortex-induced motions of the benchmark DDS over a similar parameter space [14]. For all of the simulations, a $9 B_{L} \times 6 B_{L} \times 3 B_{T}$ ( $3 B_{L}$ in front of the model and $6 B_{L}$ after the model, see Figure 3) sized computational domain was used in the present simulations (where $B_{L}$ is the overall width of the structure and $B_{T}$ is the draft of the structure). Only three degrees of freedom (DOF) on the horizontal plane (transverse, in-line and yaw motions) are allowed. In previous studies [14, 18], the mesh convergence and time step convergence have been performed and validated rigorously. The overall element mesh domain is illustrated at a mid-depth horizontal layer in Figure 3.

All the boundary conditions are kept the same in the present study. A non-slip wall boundary has been defined on the surface of the platform. At the inlet boundary, a velocity inlet is specified directly with a uniform and constant velocity. And a pressure outlet is defined on the outlet boundary. In addition, a symmetry boundary has been applied on the free surface and the two side boundaries. 
boundary has been applied on the free surface and the two side boundaries.

The present numerical simulations were performed with an overall domain contains about 7.8 million elements. A nondimensional time step $(\Delta t U / L$, where $\Delta t$ is the time step, $U$ is the inlet velocity and $L$ is the width of the DDS column) of 0.008 has been employed in the current simulations. The $y^{+}$ values are smaller than 1 in all simulations and the CFL numbers for the majority of the overall flow region are less than 1. Only in some tiny flow areas, the CFL numbers are found to be between 1 to 2 . Based on the recent study of modelling vortexinduced motions of a deep-draft semi-submersible [14, 18], the selected mesh and time step is considered to be fine enough for the current simulations' requirement.

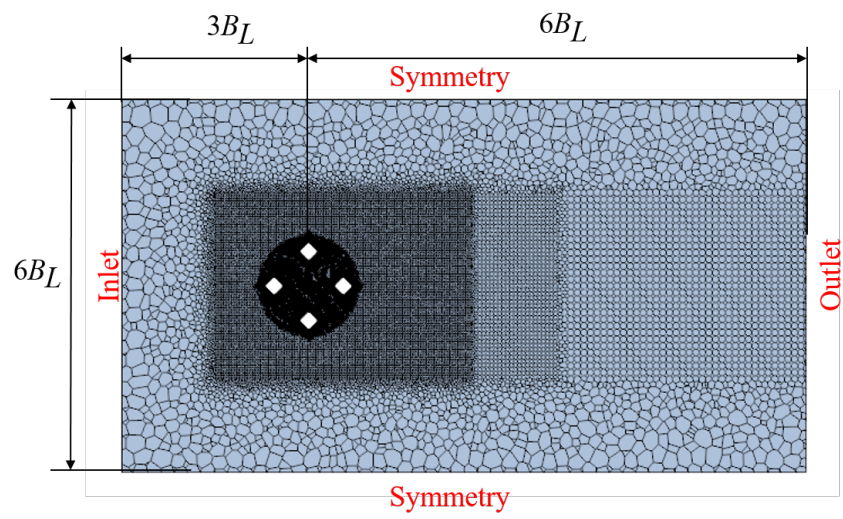

Figure 3. Visualization of the mesh at the middle draft level of the DDS (XY plane at the middle draft of the DDS).

\section{Validation study}

To validate the present numerical model, a comparison of $\bar{C}_{D}, C_{\text {Lrms }}$ and $A_{y} / L$ between the present numerical predictions with previous experimental results perform in a towing tank [14] at $U r=6.6$ is provided in Table 2. It is noted that only three degrees of freedom (namely transverse, in-line and yaw) were allowed in both experiments and numerical simulations. The motion responses of the benchmark model and hydrodynamic force on the benchmark model are compared to perform the validation study. The motion time history was recorded with a minimum of ten oscillation cycles in the transverse direction to reach the quasi-steady state of VIM. As can be seen in Table 2, all the relative differences are less than $10 \%$. Thus, a good agreement has been demonstrated between the present numerical model and the previous experimental data.

\section{RESULTS AND DISCUSSION}

The motion response of a typical moored DDS with tubercles corner under three current velocities for the headings of 45 degree were investigated using the present numerical model and their results are further compared with the previous numerical outcomes [14]. The motion measurements for more than ten cycles of the VIM oscillation period are collected in the present study.

Table 2. Comparison of the results from the present numerical model and previous experimental measurements [14] for a single platform at $U r=6.6$.

\begin{tabular}{ccll}
\hline & Num. & Exp. [14] & Relative difference (\%) \\
\hline$\overline{\boldsymbol{C}}_{\boldsymbol{D}}$ & 1.419 & 1.416 & 2.0 \\
$\boldsymbol{C}_{\boldsymbol{L r m s}}$ & 0.292 & 0.269 & 8.5 \\
$\boldsymbol{A}_{\boldsymbol{y}} / \boldsymbol{L}$ & 0.760 & 0.742 & 2.5 \\
\hline
\end{tabular}

\section{Natural period in the transverse direction}

Table 3 presents the transverse motion natural period in calm water performed by numerical simulation. As can be seen in Table 3, the tubercle design increases the transverse motion natural period slightly compared with the benchmark DDS model. Based on the previous outcomes it is observed that the inline, transverse and yaw natural frequencies are relatively close to each other. Thus, in the present study, the transverse natural period has been accepted as the natural period of reduced velocity $(U r)$.

The reduced velocity $(U r)$ is defined as:

$$
U r=\frac{\left(U T_{0}\right)}{D}
$$

where $U$ is the current speed, $T_{0}$ is the transverse natural period in calm water and $D$ is the projected width of the column (with the aim to make the results are comparable between different corner design, the project width of the column for different corner designs are set the same as a sharp square corner shape, which is $0.431 \mathrm{~m}$ ).

Table 3. The natural periods of the transverse motions in calm water.

\begin{tabular}{ll}
\hline & Transverse Natural Period $\boldsymbol{T}_{\boldsymbol{0}}(\mathbf{s})$ \\
\hline Tubercle Design & 21.3 \\
Benchmark DDS & 20.5 \\
\hline
\end{tabular}

\section{Motion characteristics}

Figure 4, Figure 5 and Figure 6, which compare the numerical results from the tubercle corner with those from the benchmark DDS model, present the non-dimensional characteristic amplitude $\left(A_{x} / L, A_{y} / L\right.$, where $U r$ is defined based on $T_{0}$ ) for motions in both the in-line and the transverse directions under the flow incidence of $45^{\circ}$. The non-dimensional amplitude is defined as $\sqrt{2} \times \sigma\left(\frac{y(t)}{L}\right)$ (where $\sigma$ is the standard deviation of the time series $y(t) / L$, and $y(t)$ represents the time series of in-line and transverse motions. For yaw motions, the non-dimensional amplitude is defined as $\sqrt{2} \times \sigma(\operatorname{yaw}(t))$. In the 
present study, the width of the column $(L)$ for both the benchmark semi and the tubercles design semi is $0.305 \mathrm{~m}$.

As can be seen in Figure 4, the tubercle design reduces the in-line motion of the structure, especially in the "lock-in" region (when $U r$ is around 7). The largest reduction is about $48 \%$ of the benchmark model in-line motion amplitude.

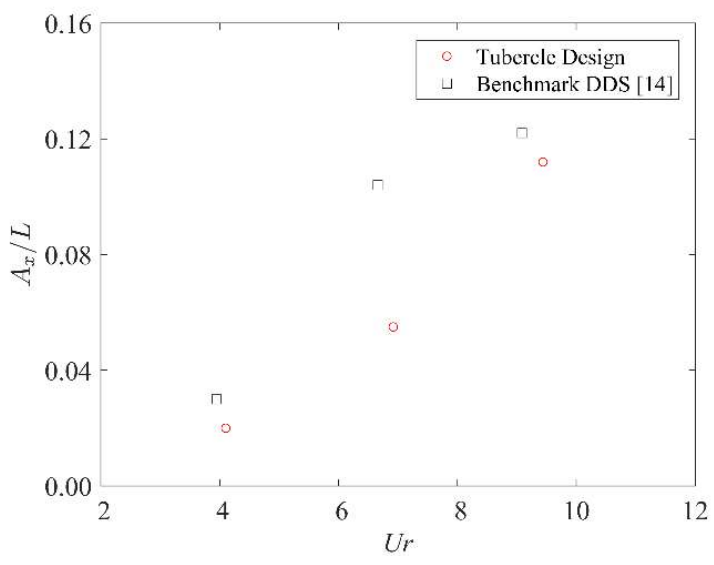

Figure 4. Non-dimensional in-line characteristics amplitudes $\left(A_{x} / L\right)$.

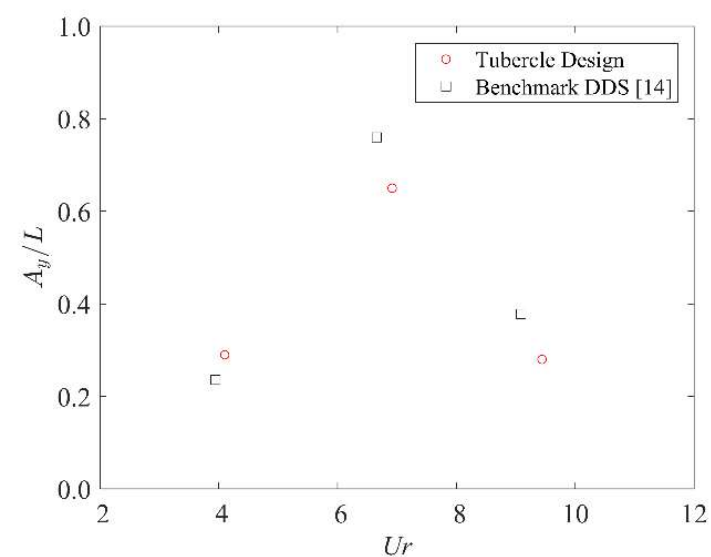

Figure 5. Non-dimensional transverse characteristics amplitudes $\left(A_{y} / L\right)$.

For the transverse motion, the tubercle design successfully reduces the motion amplitude in the "lock-in" (see Figure 7) and "post lock-in" region. However, it increases the transverse motion slightly in the "pre lock-in" region. The reduction rate in the "lock-in" region is about $15 \%$ of the benchmark model transverse motion amplitude.

As can be seen in Figure 7, the transverse motions for both model are close to a sinusoidal trajectory. The tubercle design decreases the peak value on both crest and trough.

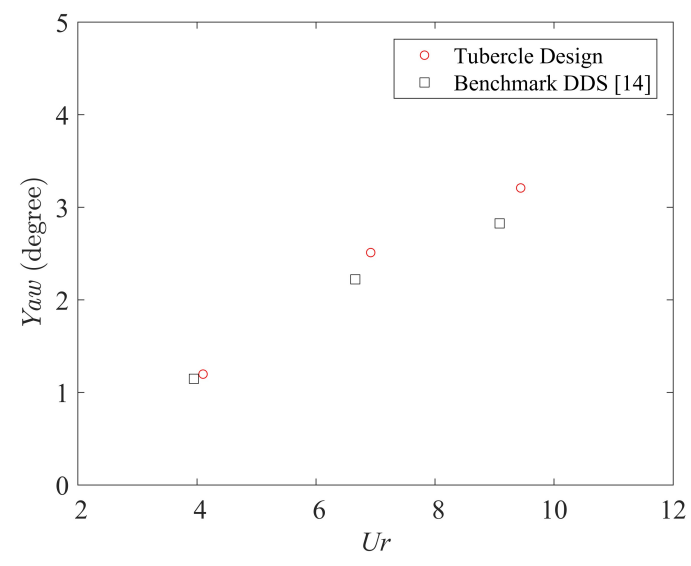

Figure 6. Non-dimensional yaw characteristics amplitudes.

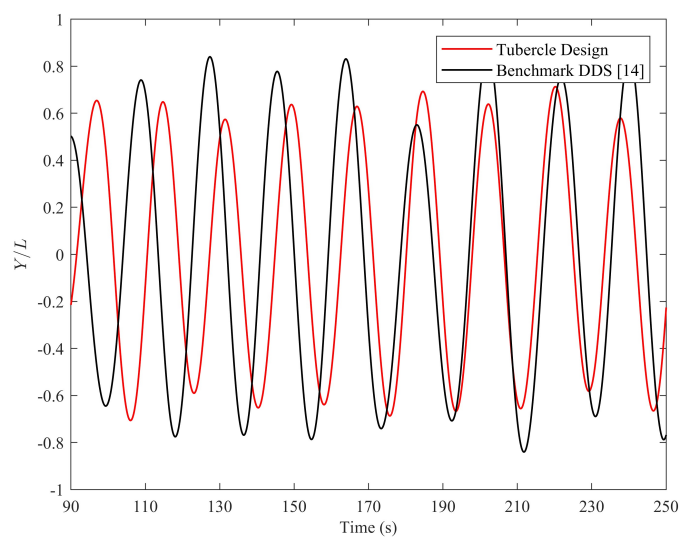

Figure 7. Time history of the transverse motion at $U r=6.7$ (benchmark); $U r=6.9$ (tubercles design).

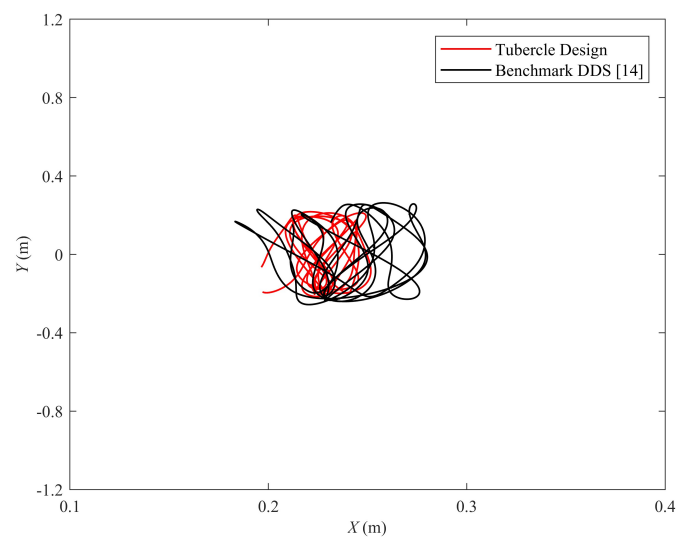

Figure 8. Motion trajectory of the platform at $U r=6.7$ (benchmark); $U r=6.9$ (tubercles design). 
However, in Figure 6, the tubercle design increases the yaw motion amplitude slightly for all cases simulated in the present study. The largest amplification rate is around 14\% (in the "post lock-in" region) of the benchmark model yaw motion amplitude.

Figure 8 , which is the motion trajectory of both platforms (the benchmark and the tubercles model in the "lock-in" region), demonstrates that the tubercle design reduces the in-line motion significantly.

It is noted that the transverse motions are somewhat sinusoidal with nearly constant amplitude as would be expected at "lock-in". However, in the "pre lock-in" and "post lock-in" region, the time history of the non-dimensional transverse amplitude shows considerable variability. Thus, the difference between the maximum and minimum amplitude, especially on the transverse direction should be compared. In the present study, the difference between the maximum and minimum transverse motion amplitude is defined as:

$$
A_{m} / L=\left(A_{\max }-A_{\operatorname{mim}}\right) / L
$$

where, $A_{\max }$ is the maximum transverse amplitude during the simulation and $A_{m i m}$ is the minimum transverse amplitude during the simulation.

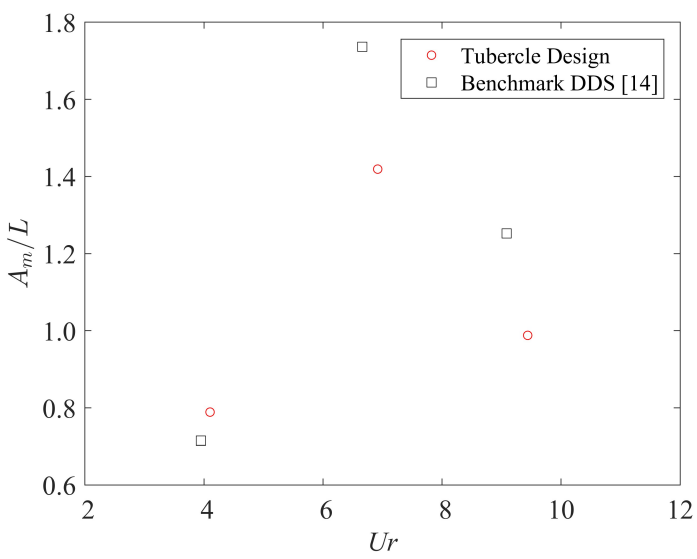

Figure 9. Non-dimensional transverse maximum difference amplitudes.

As shown in Figure 9, the tubercle design reduces the transverse motion amplitude significantly in the "lock-in" and "post lock-in" region (18\% and $21 \%$ respectively).

Thus, it can be concluded that the tubercle design is a theoretically good design for restraining VIM responses.

\section{Force analysis}

The force coefficients $\left(C_{D}, C_{L}\right)$ are the nominal coefficients describing the drag and lift forces on the structure, which are defined as:

$$
\begin{aligned}
C_{D} & =\frac{F_{D}}{\frac{1}{2} \rho U_{C}^{2} A}, \\
C_{L} & =\frac{F_{L}}{\frac{1}{2} \rho U_{C}^{2} A},
\end{aligned}
$$

where, $F_{D}$ is the drag force on the structure, $F_{L}$ is the lift force on the structure, $\rho$ is the fresh water density, $U_{C}$ is the free stream velocity, $A$ is the projected area of the immersed structure.

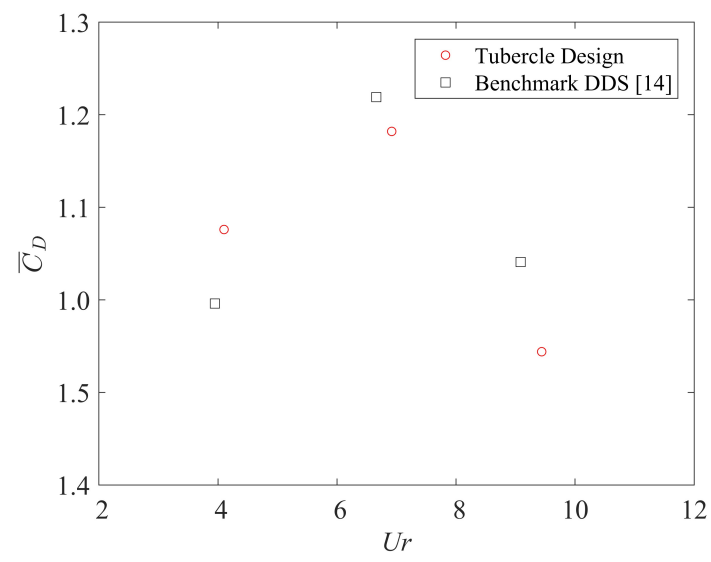

Figure 10. Mean drag coefficient $\left(\bar{C}_{D}\right)$ for the tubercle design model and the benchmark DDS.

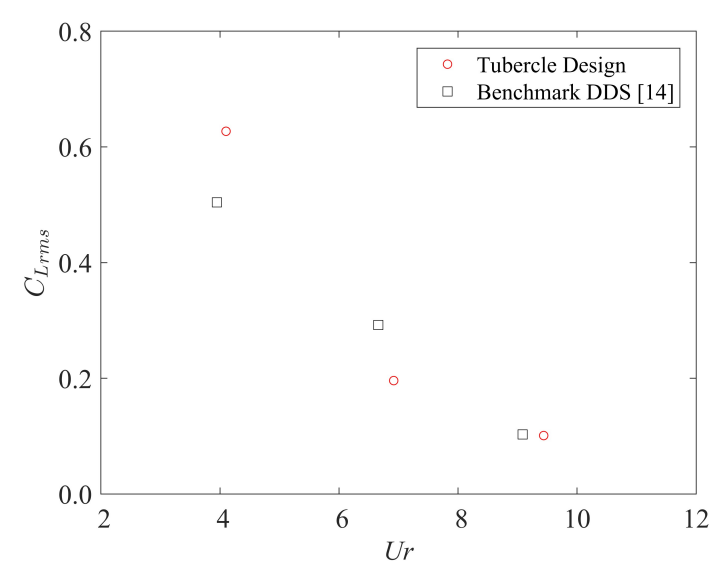

Figure 11. Root mean square lift coefficient $\left(C_{\text {Lrms }}\right)$ for the tubercle design model and the benchmark DDS

It is noted that the difference of the hydrodynamic forces on the structure between the tubercle design model and the benchmark DDS is quite similar to the motion response (see Figure 10 and Figure 11). However, in the "post lock-in" region, the root mean square lift coefficient of the two models is relatively close to each other.

\section{Flow characteristics}

To understand the vertical structures of the wake regions, a vortex identification method based on the $Q$-criterion [19] has been employed in the present study. The isofurfaces are shown at a constant positive value where $Q=1$ in Figure 12 and Figure 13. 


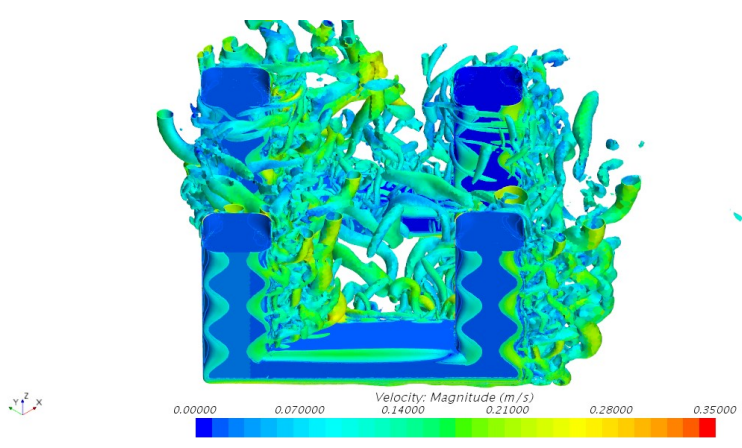

Figure 12. Isometric view representation of $Q$-criterion for the tubercle design at $U r=6.9$, when $Q=1$.

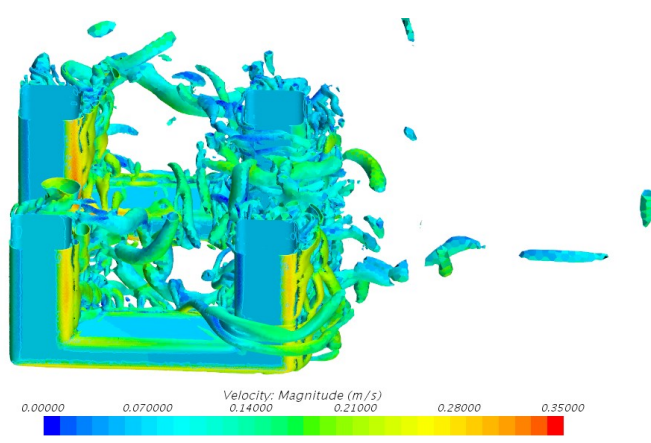

Figure 13. Isometric view representation of $Q$-criterion for the benchmark model at $U r=6.6$, when $Q=1$.

As can be seen in Figure 12, the tubercles on the corners of the column result in a continuous spanwise (column-wise) variation of the shear-layer separation points. In Figure 13, the strong vortices shed from the corner edge is broken by the tubercles in Figure 12. Thus, the vortex-induced motions causing by the resonance could be suppressed.

\section{CONCLUSIONS}

The hydrodynamic performance of the biomimetic tubercle corner design on a semi-submersible at 45 degree incidence have been numerically studied in the present work. It is demonstrated that, as the corner shape design changed, the motion responses alter drastically. The major results are summarized as:

(1) The tubercle design on a semi-submersible could reduce $15 \%$ of the non-dimensional transverse motion amplitude compared with the present benchmark DDS model in the "lockin" region.

(2) The tubercle design reduces the in-line motion of the structure, especially in the "lock-in" region. The largest reduction is about $48 \%$ of the benchmark model in-line motion amplitude

(3) The tubercles design increases the yaw motion for all the cases simulated in the present study.

(4) The numerical results showed that the three-dimensional effect, which causes spanwise flow, can be reduced by a continuous spanwise (column-wise) variation of the shear-layer separation points. Thus, the vortex-induced motions causing by the resonance could be suppressed.

(5) The present study is aimed to provide an initial idea to suppress VIM by changing the corner design. It is noted that the present tubercle design is not the best optimization design. Further study, including more tubercle designs (altering the sinusoidal corner-shape with different parameters), is suggested. In addition, the consequences of practical constructions should be considered along with the development.

\section{ACKNOWLEDGEMENTS}

Results were obtained using the ARCHIE-WeSt High Performance Computer (www.archie-west.ac.uk) based at the University of Strathclyde. 


\section{REFERENCES}

[1] Assi, G. R., and Bearman, P. W., 2018, "Vortex-induced vibration of a wavy elliptic cylinder," Journal of Fluids and Structures, 80, pp. 1-21.

[2] Darekar, R., and Sherwin, S., 2001, "Flow past a bluff body with a wavy stagnation face," Journal of Fluids and Structures, 15(3-4), pp. 587-596.

[3] Delany, N. K., and Sorensen, N. E., 1953, Low-speed drag of cylinders of various shapes, National Advisory Committee for Aeronautics.

[4] Bearman, P. W., Graham, J. M. R., Obasaju, E. D., and Drossopoulos, G. M., 1984, "The influence of corner radius on the forces experienced by cylindrical bluff bodies in oscillatory flow," Applied Ocean Research, 6(2), pp. 83-89.

[5] Tamura, T., and Miyagi, T., 1999, "The effect of turbulence on aerodynamic forces on a square cylinder with various corner shapes," Journal of Wind Engineering and Industrial Aerodynamics, 83(1), pp. 135-145.

[6] Liang, Y., and Tao, L., "Hydrodynamics Around a Deep-Draft Semi-Submersible With Various Corner Shapes," Proc. ASME 2018 37th International Conference on Ocean, Offshore and Arctic Engineering, American Society of Mechanical Engineers, pp. V009T013A001-V009T013A001.

[7] Van Nierop, E. A., Alben, S., and Brenner, M. P., 2008, "How bumps on whale flippers delay stall: an aerodynamic model," Physical review letters, 100(5), p. 054502.

[8] Weber, P. W., Howle, L. E., and Murray, M. M., 2010, "Lift, drag, and cavitation onset on rudders with leading-edge tubercles," Marine technology, 47(1), pp. 27-36.

[9] Fish, F. E., Weber, P. W., Murray, M. M., and Howle, L. E., 2011, "The tubercles on humpback whales' flippers: application of bio-inspired technology," Oxford University Press.

[10] Isaac, K., and Swanson, T., "Biologically inspired wing leading edge for enhanced wind turbine and aircraft performance," Proc. 6th AIAA Theoretical Fluid Mechanics Conference, p. 3533.

[11] Corsini, A., Delibra, G., and Sheard, A. G., 2013, "On the role of leading-edge bumps in the control of stall onset in axial fan blades," Journal of Fluids Engineering, 135(8), p. 081104.

[12] Shi, W., Atlar, M., Norman, R., Aktas, B., and Turkmen, S., 2016, "Numerical optimization and experimental validation for a tidal turbine blade with leading-edge tubercles," Renewable Energy, 96, pp. 42-55.

[13] Shi, W., Atlar, M., Norman, R., Day, S., and Aktas, B., 2019, "Effect of waves on the leading-edge undulated tidal turbines," Renewable energy, 131, pp. 435-447.

[14] Liang, Y., Tao, L., Xiao, L., and Liu, M., 2017, "Experimental and numerical study on vortex-induced motions of a deep-draft semi-submersible," Applied Ocean Research, 67, pp. 169-187.

[15] Shur, M. L., Spalart, P. R., Strelets, M. K., and Travin, A. K., 2008, "A hybrid RANS-LES approach with delayed-DES and wall-modelled LES capabilities," International Journal of Heat and Fluid Flow, 29(6), pp. 1638-1649.
[16] Spalart, P. R., Jou, W. H., Strelets, M., and Allmaras, S. R., 1997, "Comments on the feasibility of LES for wings, and on a hybrid RANS/LES approach," Advances in DNS/LES, 1, pp. 48 .

[17] CD-adapco, 2018, "User Guide," Star-CCM+ Version 13.04.011.

[18] Liang, Y., and Tao, L., 2017, "Interaction of vortex shedding processes on flow over a deep-draft semi-submersible," Ocean Engineering, 141, pp. 427-449.

[19] Hunt, J. C., Wray, A. A., and Moin, P., 1988, "Eddies, streams, and convergence zones in turbulent flows." 


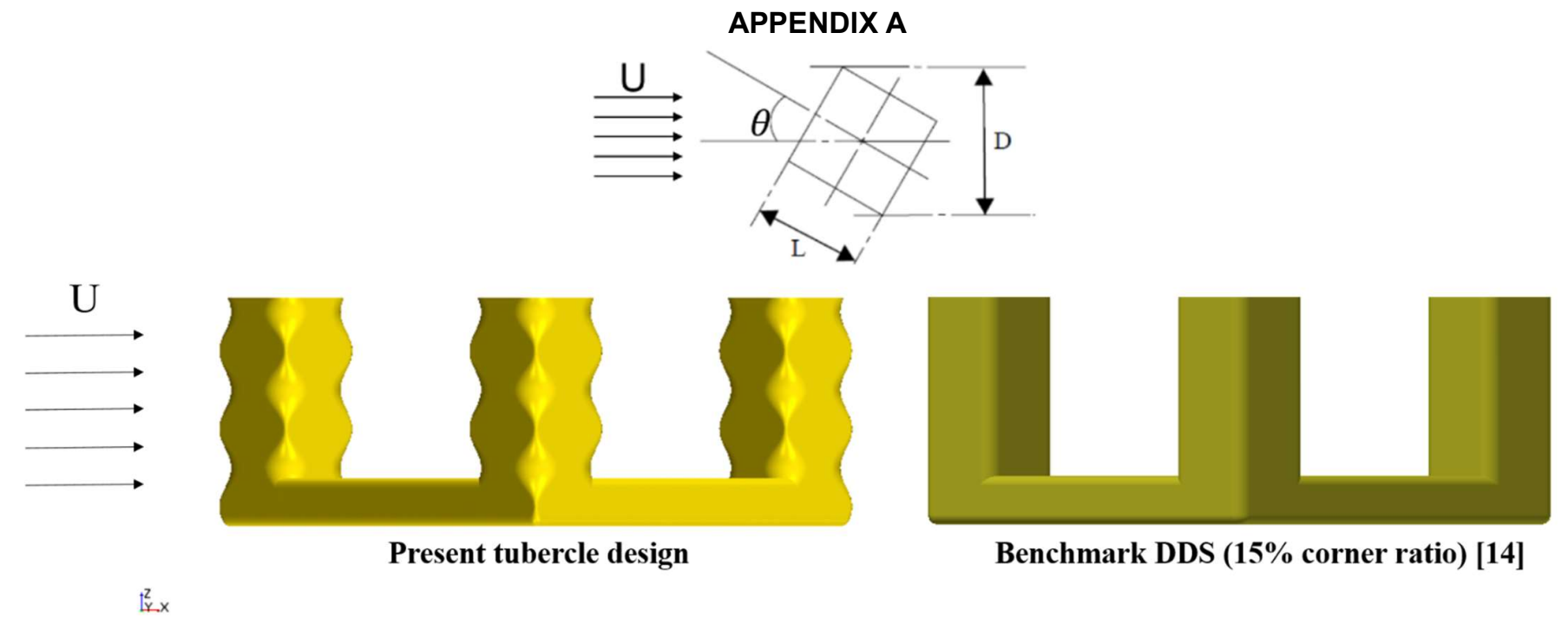

Figure 14. Present tubercle design.

Table 4. Sinusoidal corner-shape/Tubercles Design (Where $Z$ is the distance from the baseline of the platform; $Z=0.367 \mathrm{~m}$ is the water level).

\begin{tabular}{ccc}
\hline $\boldsymbol{Z}$ & corner radius (m) & corner ratio \\
\hline $\mathbf{0 . 0 0}$ & 0.052 & 0.170 \\
$\mathbf{0 . 0 5}$ & 0.008 & 0.027 \\
$\mathbf{0 . 1 0}$ & 0.025 & 0.082 \\
$\mathbf{0 . 1 5}$ & 0.079 & 0.258 \\
$\mathbf{0 . 2 0}$ & 0.095 & 0.313 \\
$\mathbf{0 . 2 5}$ & 0.052 & 0.170 \\
$\mathbf{0 . 3 0}$ & 0.008 & 0.027 \\
$\mathbf{0 . 3 5}$ & 0.025 & 0.082 \\
$\mathbf{0 . 4 0}$ & 0.079 & 0.258 \\
$\mathbf{0 . 4 5}$ & 0.095 & 0.313 \\
$\mathbf{0 . 5 0}$ & 0.052 & 0.170 \\
$\mathbf{0 . 5 5}$ & 0.008 & 0.027 \\
$\mathbf{0 . 6 0}$ & 0.025 & 0.082 \\
$\mathbf{0 . 6 5}$ & 0.079 & 0.258 \\
$\mathbf{0 . 7 0}$ & 0.095 & 0.313 \\
$\mathbf{0 . 7 3 4}$ & 0.070 & 0.229 \\
\hline
\end{tabular}




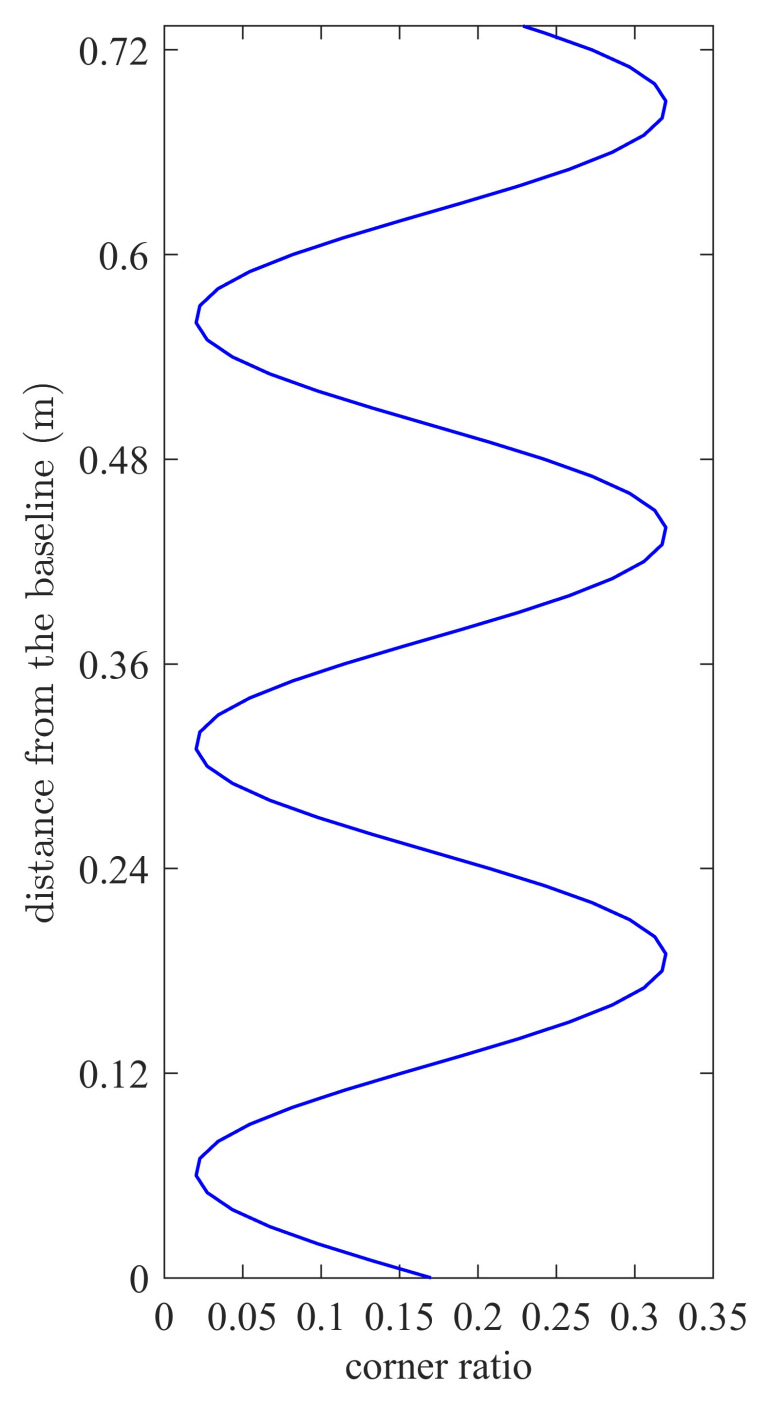

Figure 15. Sinusoidal corner-shape/Tubercles Design. 